\title{
Family functioning and mental wellbeing impairment during initial quarantining for the COVID-19 pandemic: A study of Canadian families
}

\author{
Philippe Hwang $^{1}$ (D) Lara Ipekian $^{1} \cdot$ Nikhil Jaiswal $^{1} \cdot$ Gabie Scott $^{1} \cdot$ Evangelina Lila Amirali $^{1} \cdot$ Lily Hechtman $^{1,2}$
}

Accepted: 29 December 2021

(c) The Author(s), under exclusive licence to Springer Science+Business Media, LLC, part of Springer Nature 2022

\begin{abstract}
Quarantine measures imposed due to COVID-19 have negatively impacted individual wellbeing. However, the research on the factors impacting mental health and functioning of families is limited. The current study explores socio-economic and demographic factors that mediate poor family functioning, anxiety, and depressive symptoms in response to quarantine measures in Canadian parents and children. 254 Canadian families completed an online questionnaire capturing demographic information and mental wellbeing of individuals and of the whole family. Family functioning was assessed using the Family Assessment Device General Functioning subscale (FAD-GF), and individual mental wellbeing was measured with the Generalized Anxiety Disorder screener (GAD-7) and Patient Health Questionnaire (PHQ-9). Generalized linear models and logistic regression were used to model socio-demographic impacts on outcome variables. Problematic family functioning was found in $78.5 \%$ of families with a high proportion of parents reporting above-threshold symptoms of anxiety (62.9\%) and depression (73.4\%). Many children also reported above-threshold symptoms of anxiety (54.6\%) and depression (70.7\%). Family functioning was impacted by parent and child age, parental employment status and pre-existing conditions for children. Anxiety and depression experienced by parents and children was increased in families with parents aged $<45$, household income $<\$ 100,000$ pre-existing psychiatric conditions, or having a child aged $>5$ years. These findings show that most Canadian families observed in this study experienced above threshold symptoms of anxiety, depression and poor family functioning. Our study provides an initial step towards identifying characteristics of at-risk families and targeting interventions to mediate negative effects of quarantining.
\end{abstract}

Keywords COVID-19 $\cdot$ families $\cdot$ depression $\cdot$ anxiety $\cdot$ children $\cdot$ parents

\section{Introduction}

The COVID-19 pandemic resulted in quarantine measures restricting work, leisure, and school activities, severely impacting the mental wellbeing of individuals and families (Torales et al., 2020). During the initial quarantine period there was a complete lockdown put in place in Canada. Schools were closed and most people were working from home because businesses and other enterprises were closed.

Philippe Hwang

Philippe.hwang@mail.mcgill.ca

1 Department of Child Psychiatry, The Montreal Children's Hospital, Research Institute of the McGill University Health Centre, Room A04.4205, 1001 Boulevard Décarie, Montréal, QC H4A 3J1, Canada

2 Department of Child and Adolescent Psychiatry, McGill University, Montreal, Canada
Brooks et al. (2020) conducted a meta-analysis on the mental wellbeing of laypeople and healthcare workers during virus outbreaks between 2004 and 2016, specifically during SARS, H1N1, Ebola and MERS outbreaks. According to this synthesis, distress symptoms associated with quarantine measures put in place due to virus outbreaks include anxiety and depressive symptoms such as anger, low mood, irritability, confusion and exhaustion. The authors suggest short quarantine periods, keeping people well-informed about the virus, ensuring basic needs and improving virtual communication to reduce adverse mental health effects (Brooks et al., 2020). Similarly, several reviews have examined studies in COVID-19 patients, patients with medical and psychiatric illnesses, and the general public. Findings revealed increased anxiety and depressive symptoms with pooled prevalence rates between $28 \%$ - 33\% for each symptom grouping (Vindegaard \& Benros, 2020; Salari et al., 2020; Luo et al., 2020). However, few studies have examined the 
impact of the pandemic and isolation measures, specifically on families.

The review by Luo et al. (Luo et al., 2020) notes several risk factors for more severe psychological impact due to COVID-19 found in several studies, including being female, being in situations that increase COVID-19 risk (e.g. contact with COVID-19 patients, poorer health), lower socio-economic status (unstable income, lower education, rural location), and social isolation. They suggest that family support, precautionary measures, and good healthcare infrastructure including resources and up-to-date information may act as protective factors. These findings mirror those of Vindegaard and Benros (Vindegaard \& Benros, 2020) who also associate sociodemographic factors with anxiety and depression during the pandemic including education level, number of children, urban and rural location. They also discuss the association of pre-existing medical and psychiatric conditions with increased risk of depression (Mazza et al., 2020; Wang et al., 2020; Zhang et al., 2020; Li et al., 2020) and/ or anxiety (Mazza et al., 2020; Wang et al., 2020; Zhang et al., 2020; Li et al., 2020; Özdin \& Bayrak Özdin, 2020). (Mazza et al., 2020; Wang et al., 2020; Zhang et al., 2020; Li et al., 2020; Özdin \& Bayrak Özdin, 2020). A positive relationship exists between involuntarily working from home and strain surrounding work-family conflict (Lapierre et al., 2016). Altered daily living conditions and family environments caused by the pandemic and quarantine measures may add a new dimension of difficulties or exacerbate existing ones for individuals, placing strain on the family unit (Prime et al., 2020).

\section{Family Functioning}

The impact of COVID-19 on family wellbeing and functioning has not been widely documented, but preliminary studies have shown increasing parental stress due to the pandemic (Miller et al., 2020; Davidson et al., 2020). The scientific community has drawn from evolving literature on the effects of quarantining on children and adults (Prime et al., 2020; Miller et al., 2020; Davidson et al., 2020), and from studies on previous pandemic and disaster situations (Sprang \& Silman, 2013; Markenson \& Redlener, 2004; Nicholas et al., 2008) to examine the potential impacts on family functioning. Furthermore, younger age, greater number of home stressors and poor supervisor family-supportive behaviours may lead to negative mental health outcomes for adults unexpectedly transferred to working from home (Evanoff et al., 2020). The primary sources of strain within families due to COVID-19 quarantine measures may come from increased economic and organizational stressors placed on parents or caregivers and disruptions to usual schedules and organization for all members (Prime et al., 2020; Davidson et al., 2020; Stark et al., 2020). These stressors may erode existing adaptive processes, worsening the family wellbeing as well as individuals' mental health.

This study examines the socio-economic and demographic factors that mediate poor family functioning and increase the likelihood of experiencing anxiety and depression symptoms for Canadian families during the beginning of the COVID-19 pandemic. Our specific goals were: (1) to identify the factors predicting increased anxiety and depressive symptoms in families; and (2) to identify the factors that increase the odds of having problematic family functioning for families.

\section{Materials and Methods}

\section{Study Design}

This cross-sectional study collected data from Canadian families between May 21 and June 04, 2020. Eligibility criteria included (i) currently living in Canada, (ii) having at least one child aged 18 or younger, (iii) parents older than 18, (iv) being able to read and understand either English or French and (v) having internet access. The survey was conducted online via JotForm (HIPAA-compliant form service) and participants were informed of the study via social media advertisements, referrals from other participants, and parent associations with a survey link made available in all advertisements. Participants read, digitally consented, then completed the self-administered online survey. Valid participants were offered a \$10 CAD Amazon gift card following completion of the survey. To evaluate the potential risks and effects of quarantining on the mental health of children, and better understand the factors that might worsen the mental health in these families, the child who was most impacted by quarantine measures or whose mental wellbeing was most affected by the pandemic was sought. However, information concerning one child and the respondent parent in the household was sought to decrease the burden for the family and make the participants more receptive to completing study questionnaires. The study was reviewed and approved by the McGill University Health Center Research Institute's Ethics Review Board. The consent form encouraged participants to seek counsel from a health care professional if they exhibited symptoms of anxiety, depression, or poor family functioning.

\section{Study Instruments}

This study invited the respondent parent and the child most impacted by pandemic isolation measures to respond to questions regarding (a) family socio-economic demographic 
information, (b) symptoms of anxiety, depression, and family functioning.

Socio-economic and demographic information included: age, sex, ethnicity, marital status, highest education attained and parents' employment status, annual household income (Canadian Dollars (CAD)), and whether these last two had been impacted by the pandemic. Parents provided their age according to the following categories: 18-24, 25-34, 35-44, $45-54,55-64,65-74,75+$, in order to reduce potential participant attrition due to reluctance with sharing their precise age. For children, the exact age was requested. However, the child age categories used for analysis (0-5, 6-9, 10-13, and 14-18) were selected according to the following priorities: (1) differentiating between children who were definitely preschool (0-5) and school-aged (6+), (2) children who were definitely in elementary or middle school (6-13) and those in high school (14-18), (3) attempting to maintain a rough balance of years of age between groups (4-5 years). Participants were asked about pre-existing medical and psychiatric conditions in the parents and children. Pre-existing psychiatric conditions included attention-deficit hyperactivity disorder, bipolar disorder, depression, anxiety, schizophrenia, and substance abuse. Participants were given the option to indicate unlisted conditions. Participants were also asked about pre-existing medical conditions (heart, lung and breathing, diabetes, gastrointestinal disease, cancer, autoimmune conditions, and liver disease).

The Generalized Anxiety Disorder-7 (GAD-7)(Spitzer et al., 2006) scale was used to measure self-reported anxiety symptom severity over a two-week recall period on 7 items rated on a 4-point scale ( $0=$ "not at all", $1=$ "several days", $2=$ "more than half the days", and $3=$ "nearly every day"). Total scores range from 0 -21, with a total score of $\geq 10$ indicating possible anxiety (Spitzer et al., 2006; Löwe et al., 2008). Löwe et al. (2008) report good internal consistency for the GAD-7 ( $\alpha=0.89$ ) (Löwe et al., 2008). Scores are categorized "minimal anxiety" (0-4), "mild anxiety" (5-9), "moderate anxiety" (10-14) and "severe anxiety" (15-21).

The Patient Health Questionnaire (PHQ-9)(Kroenke et al., 2001) was used to assess symptoms of depression. Response options were $0=$ "not at all", $1=$ "several days", $2=$ "more than half the days" and 3="nearly every day" and recall of symptoms was requested over a two-week period. Scores range from $0-27$, with a total score of $\geq 10$ indicating possible major depression (Kroenke et al., 2001; Manea et al., 2012). Kroenke, Spitzer \& Williams (2001) report excellent internal reliability for the PHQ-9 $(\alpha=0.89)$ (Kroenke et al., 2001). Scores are categorized "minimal depression" (0-4), "mild depression" (5-9), "moderate depression" (10-14), "moderately severe depression" (15$19)$ and "severe depression" (20-27).

At the time of the study, schools across Canada were closed, and children were receiving education remotely.
Families filling out the survey were instructed to have children complete the questionnaires themselves. It is important to note that the GAD-7 and the PHQ-9 are not validated for children under 12. However, to obtain an impression of the mental wellbeing of the younger children, parents of children in this age group (73\%) were encouraged to assist with responding to these questionnaires to provide some impression of the child's anxious and depressive symptoms. We recognize the limitation of this approach and discuss this further in the study limitations. In contrast, children above $12(27 \%)$ were encouraged to complete the survey alone.

The Family Assessment Device General Functioning Subscale (FAD-GF) is a 12-item self-report measure of family functioning, a subset of the 60-item McMaster Family Assessment Device (FAD)(Epstein et al., 1983) which identifies families experiencing difficulties. Responses are scored on a 4-point Likert scale from $1=$ "strongly agree" to $4=$ "strongly disagree". A mean of these scored responses represents an individual's score. Both the FAD and the FAD-GF have been widely used in clinical and general populations with a mean of 2.0/4.0 on the FAD-GF. The FAD has been reported as having good diagnostic reliability $(83 \%)$, sensitivity $(67 \%)$ and specificity $(64 \%)$.(Miller et al., 1985; Staccini et al., 2015) Questions assess multiple categories including problem-solving, communication, and decision-making.

\section{Statistical Analysis}

Data was extracted from JotForm onto MS Excel, dummycoded (e.g. $1=$ female, $2=$ male), and exported to SPSS for analysis (IBM, 2017). Descriptive statistics of the sample's factor and outcome variables were reported. Pre-existing medical and psychiatric conditions in children and parents were individually summed and categorized.

Univariate general linear models with ANOVA-F testing were used to identify the factors impacting outcome variables of anxiety and depression. Factors that significantly affected outcome measures were further investigated for pairwise mean differences and were adjusted using Bonferroni correction to control for multiple comparisons (Miller, 1966). Estimated marginal means for anxiety and depression were derived by the statistical model for each factor, adjusting for all other factors, to describe overall and pairwise contributions to the variance. A binary logistic regression model using backward likelihood ratio selection was fitted with grouping variables to identify factors impacting the odds of problematic family functioning.

Listwise deletion was conducted during both binary logistic regression and univariate linear modelling to satisfy model conditions. Over $80 \%$ of participants (>207/254) were retained in each model for analysis. 


\section{Outcome Variables}

When modelling anxiety and depression, sum scores from each scale were treated as numerical values to evaluate the factors which might influence elevated anxiety and depressive symptoms in family members. In order to account for differences in perception of family functioning, individual (parent and child) scores within a family were averaged in order to obtain a whole-family functioning score, which was then binarily coded $(<2=$ non-problematic; $\geq 2=$ problematic).

\section{Grouping Variables}

For the regression modelling, parent age and sex, child age and sex, family ethnicity, annual household income, household income and employment status (and change in these due to COVID-19) as well as pre-existing medical conditions, pre-existing anxiety, depression and other psychiatric disorders for both parents and children were included. 97.2\% of parents were married/co-habiting, therefore it is assumed that both parents would be involved in child-rearing. As such, parent sex was excluded from analyses of child anxiety and depression outcomes.

\section{Results}

305 families from across Canada participated in the survey. 51 families were removed (based on exclusion criteria, duplicate submissions, or $>25 \%$ information missing). The remaining 254 families were included in modelling analysis of factors that might influence anxiety and depression symptoms, and family functioning. Model conditions required listwise deletion, but still retained over $80 \%$ of the 254 participants (Figure 1).

\section{Sample Demographics}

Sample characteristics are provided in Table 1. Proportions were computed based on valid observations among all families. For each individual variable, less than $2 \%$ of data was missing in the sample. Participants were mostly 35-44 years old (59.8\%), married, of white ethnicity, in 3-person families (two parents and one child), and the majority had a child aged less than 14 (90.9\%). Notably, families experienced high work loss due to the pandemic $(77.3 \%)$ despite no change in household income (65.9\%).

\section{Mental Health Outcomes}

The cut-off score of $\geq 10$ on the GAD-7 and PHQ-9 correspond to clinically significant anxiety and depressive symptoms, respectively. All parents and 99\% (251/254) of children fully completed the GAD-7, with $62.9 \%$ of parents and $54.6 \%$ of children scoring above threshold. All parents and $95 \%$ (242/254) of children fully completed the PHQ-9, and $73.4 \%$ of the parents and $70.7 \%$ of the children scored above the threshold of 10 on the scale.

After taking a mean of parent and child family functioning scores to represent a whole-family score, $78.5 \%$ of the participant families were above the threshold of 2.0/4.0 suggesting problematic family functioning.

\section{Factors Impacting Anxiety During Quarantine}

Univariate generalized linear modelling was performed with grouping variables outlined in 2.3.2 for parent and child anxiety scores. Pre-existing child psychiatric conditions were grouped into a single yes/no variable for parent analyses, and conversely parent psychiatric conditions were grouped for child analyses. For factors involving 3 or more groups, Bonferroni corrections for multiple comparisons were used and the resulting $\mathrm{p}$-value is presented for withingroup comparisons.

\section{Parental Anxiety}

Based on the model, the estimated marginal mean for parent's anxiety was 10.100 ( $\mathrm{SE}=1.467)$. The factors significantly impacting parents' anxiety scores were household income, parent age, sex, pre-existing anxiety, and other psychiatric conditions [Table 2].

Parent anxiety scores significantly decreased as household income increased $(\mathrm{F}(2,184)=4.28, \mathrm{p}=0.015)$. Participants with household incomes $>\$ 100,000(\mathrm{~m}=8.930$, $\mathrm{SE}=1.498$ ) had significantly lower anxiety scores than those who reported $\$ 60,000$ to $\$ 99,999(\mathrm{~m}=10.263, \mathrm{SE}=1.455$, $\mathrm{p}=0.001)$ and less than $\$ 60,000(\mathrm{~m}=11.105, \mathrm{SE}=1.646$, $\mathrm{p}=0.004)$. Parent anxiety scores significantly decreased as age increased $(\mathrm{F}(2,184)=11.33, \mathrm{p}<0.001)$, with those in the $45+$ age group $(m=6.238, \mathrm{SE}=1.590)$ having significantly lower anxiety average scores than those in the 35-44 group $(\mathrm{m}=11.746, \mathrm{SE}=1.574, \mathrm{p}<0.001)$ and the $18-34$ group $(\mathrm{m}=12.314, \mathrm{SE}=1.628, \mathrm{p}<0.001)$. Female participants $(m=10.741 \mathrm{SE}=1.541)$ had significantly higher anxiety mean scores compared to males $(\mathrm{m}=9.458, \mathrm{SE}=1.439$, $\mathrm{F}(1,184)=5.914, \mathrm{p}=0.016)$. Parents with pre-existing anxiety had significantly higher anxiety mean scores $(m=10.921$, $\mathrm{SE}=1.575)$ than those who reported no pre-existing anxiety $(\mathrm{m}=9.278, \mathrm{SE}=1.432, \mathrm{~F}(1,184)=5.997, \mathrm{p}=0.015)$. Those with other pre-existing psychiatric conditions $(m=11.301, \mathrm{SE}=1.562)$ had significantly higher anxiety scores than those with no pre-existing psychiatric conditions $(\mathrm{m}=8.898, \mathrm{SE}=1.462, \mathrm{~F}(1,184)=10.54, \mathrm{p}=0.001)$. A change 


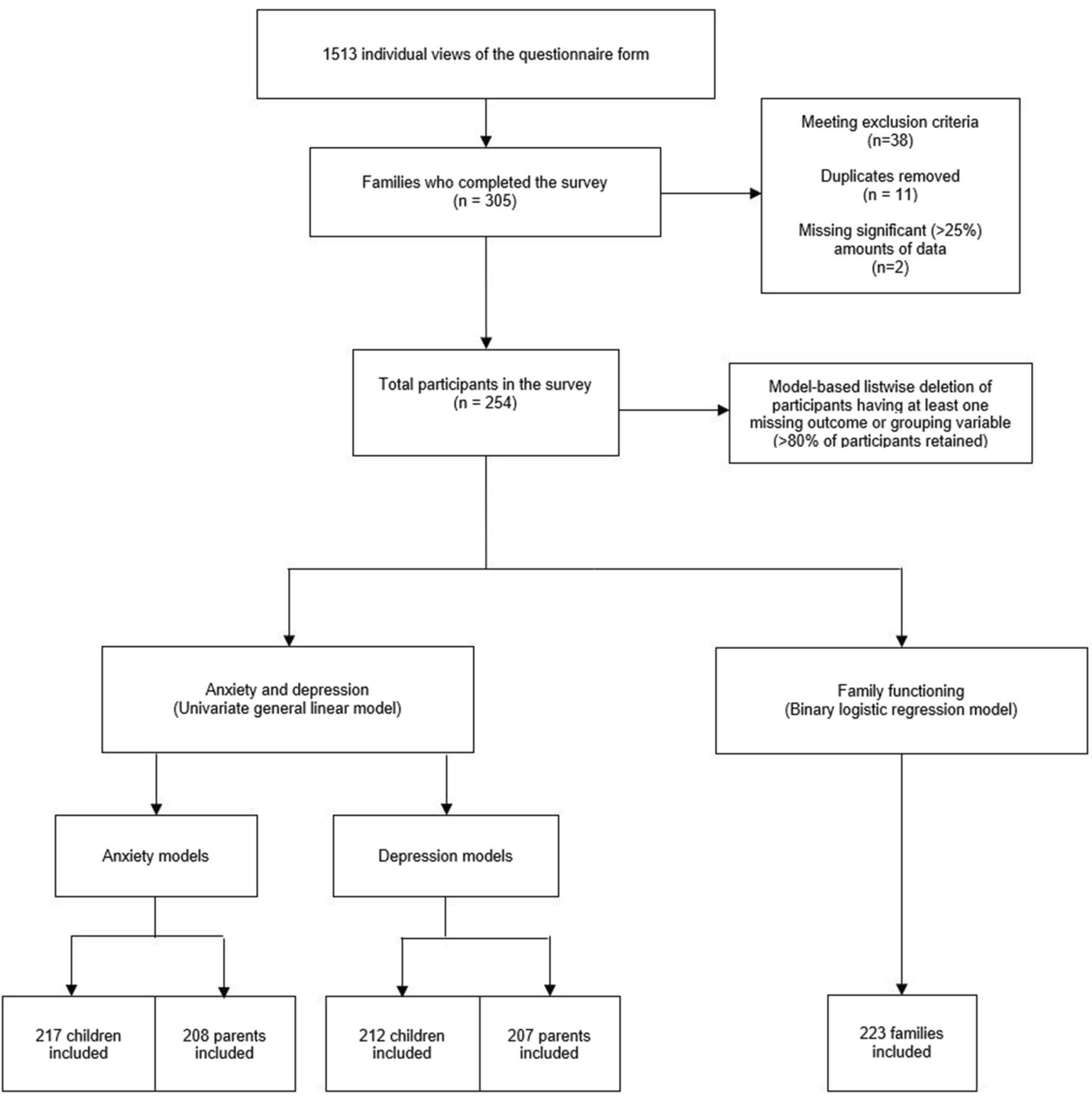

Fig. 1 Flow diagram of participant families in the study. Participants initially excluded either did not meet all the eligibility criteria, submitted duplicate responses erroneously, or were missing greater than $25 \%$ of responses. To satisfy modelling conditions, listwise deletion

in household employment or the presence of pre-existing depression in a parent did not impact anxiety scores.

Therefore, we may infer that the female parents, aged 18-44, who reported household incomes of less than $\$ 100,000$, or who reported either pre-existing anxiety or other non-depression psychiatric conditions were more vulnerable to have elevated anxiety symptom scores during quarantine. occurred for participants with any missing outcome or grouping variable for each model. Over $80 \%$ of participants were retained for each model

\section{Child Anxiety}

Based on the model, the estimated marginal mean for child anxiety was $5.036(\mathrm{SE}=1.736)$. The factors significantly impacting children's anxiety scores were household income, parent age and child age [Table 3].

Children with household incomes in the " $>\$ 100,000$ " category $(\mathrm{m}=4.031, \mathrm{SE}=1.732)$ had lower anxiety scores 
Table 1 Participant demographic information. ${ }^{\mathrm{a}}$
Participant families $(\%)^{\mathrm{b}}$

Parent Age

$18-34$

$84(33.1 \%)$

$35-44$

$152(59.8 \%)$

$45+$

$18(7.1 \%)$

Parent Sex

Male

$134(52.8 \%)$

Female

$117(46.1 \%)$

Marital Status

Married / Co-Habiting

$247(97.2 \%)$

Single, Separated, or Divorced

$7(2.8 \%)$

Ethnicity

White

$201(79.1 \%)$

Non-White

$53(20.9 \%)$

Household Size

2

3

4

$5+$

$6(2.4 \%)$

$177(69.7 \%)$

$48(18.9 \%)$

$23(9.1 \%)$

Child Age (most impacted by pandemic isolation measures)

$0-5$

$15(6.1 \%)$

$6-9$

$120(48.8 \%)$

$10-13$

$87(34.1 \%)$

$14-18$

$24(9.4 \% \%)$

Annual Household Income (CAD)

$<\$ 60,000$

$32(12.6 \%)$

$\$ 60,000-\$ 99,999$

$121(47.6 \%)$

$>\$ 100,000$

$101(39.8 \%)$

Pre-COVID Pandemic Employment Status

$221(87.7 \%)$

Employed Full-Time

$221(87.7 \%)$

Employed Part-Time

$3(1.2 \%)$

$8(3.2 \%)$

Student/Homemaker

$57(22.7 \%)$

$194(77.3 \%)$

Reduction in Hours (including job loss)

$168(65.9 \%)$

No Change/Increase

$86(33.7 \%)$

Pre-Existing Conditions - Parent

Medical Condition

$135(53.1 \%)$

Psychiatric Condition (any)

$131(51.6 \%)$

Anxiety

$62(24.4 \%)$

$29(11.4 \%)$

Pre-Existing Conditions - Children

Medical Condition

$102(40.2 \%)$

$114(44.9 \%)$

$37(14.6 \%)$

$14(5.5 \%)$

Depression

${ }^{a}$ Number of families in each category is reported based on valid observations. Less than $2 \%$ of data was missing from each variable.

${ }^{\mathrm{b}}$ Total values may not add up to 254 due to missing entries. Percentages are adjusted for valid observations

${ }^{\mathrm{c}}$ Represent a subgroup of the total with a psychiatric condition 
Table 2 Pairwise comparisons of factors impacting parent anxiety $(\mathrm{N}=208)$ and depression $(\mathrm{N}=207)$ in univariate general linear models with model-adjusted estimated marginal means ${ }^{\mathrm{a}}$

\begin{tabular}{|c|c|c|c|c|}
\hline & GAD-7 Mean (SE) & Main Effect ${ }^{b}$ & PHQ-9 Mean (SE) & Main Effect ${ }^{b}$ \\
\hline Parent Age & & $\mathrm{F}(2,184)=11.33 * * *$ & & $\mathrm{~F}(2,183)=8.38 * * *$ \\
\hline $18-34$ & $12.314(1.628)$ & & $11.604(2.070)$ & \\
\hline $35-44$ & $11.746(1.574)$ & & $9.769(2.006)$ & \\
\hline $45+$ & $6.238(1.590)$ & & $4.645(2.084)$ & \\
\hline Parent Sex & & $\mathrm{F}(1,184)=5.91^{*}$ & & $\mathrm{~F}(1,183)=12.89 * * *$ \\
\hline Male & $9.458(1.439)$ & & $7.469(1.844)$ & \\
\hline Female & $10.741(1.541)$ & & $9.876(1.982)$ & \\
\hline Child Age & & $F(3,184)=1.66$ & & $\mathrm{~F}(3,183)=2.73^{*}$ \\
\hline $0-5$ & $9.903(1.834)$ & & $6.426(2.345)$ & \\
\hline $6-9$ & $11.060(1.502)$ & & $10.250(1.927)$ & \\
\hline $10-13$ & $9.817(1.493)$ & & $8.896(1.902)$ & \\
\hline $14-18$ & $9.619(1.658)$ & & $9.119(2.140)$ & \\
\hline Household Income (CAD) & & $\mathrm{F}(2,184)=4.28^{*}$ & & $\mathrm{~F}(1,183)=3.01$ \\
\hline$<\$ 60,000$ & $11.105(1.646)$ & & $8.909(2.084)$ & \\
\hline$\$ 60,000-\$ 99,999$ & $10.263(1.455)$ & & $9.401(1.869)$ & \\
\hline$>\$ 100,000$ & $8.930(1.498)$ & & $7.708(1.934)$ & \\
\hline Change in household employment & & $F(3,184)=0.718$ & & $F(3,183)=1.018$ \\
\hline Both parents lost work hours & $10.715(1.462)$ & & $9.287(1.877)$ & \\
\hline Participant parent lost work hours & $9.950(1.568)$ & & $9.127(2.012)$ & \\
\hline Spouse lost work hours & $9.965(1.541)$ & & $8.154(1.975)$ & \\
\hline Neither parent lost work hours & $9.768(1.546)$ & & $8.123(1.877)$ & \\
\hline Parent Pre-Existing Psychiatric Condition (Anxiety) & & $\mathrm{F}(1,184)=6.00^{*}$ & & $\mathrm{~F}(1,183)=2.20$ \\
\hline Yes & $10.921(1.575)$ & & $9.295(2.023)$ & \\
\hline No & $9.278(1.432)$ & & $8.051(1.834)$ & \\
\hline Parent Pre-Existing Psychiatric Condition (Depression) & & $\mathrm{F}(1,184)=2.18$ & & $\mathrm{~F}(1,183)=1.82$ \\
\hline Yes & $10.690(1.596)$ & & $9.366(2.048)$ & \\
\hline No & $9.509(1.441)$ & & $7.980(1.855)$ & \\
\hline Parent Pre-Existing Psychiatric Condition (Other) & & $\mathrm{F}(1,184)=10.54 * * *$ & & $\mathrm{~F}(1,183)=6.39 *$ \\
\hline Yes & $11.301(1.562)$ & & $9.878(2.010)$ & \\
\hline No & $8.898(1.462)$ & & 7.467 (1.876) & \\
\hline
\end{tabular}

${ }^{a}$ Means presented are based on the estimated marginal means of the model. Bonferroni Correction applied as appropriate for multiple comparisons in individual factor ANOVA tests for subgroup differences.

${ }^{\mathrm{b}}$ Main effect of each factor presented as F-statistic [degrees of freedom(factor), degrees of freedom(error)]

$\mathrm{CAD}=$ Canadian Dollar

$\mathrm{GAD}=$ Generalized Anxiety Disorder Scale

PHQ = Patients' Health Questionnaire (depression scale)

$\mathrm{SE}=$ Standard Error

$* \mathrm{p}<0.05$

$* * \mathrm{p}<0.01$

$* * * \mathrm{p}<0.001$

$(\mathrm{F}(2,194)=5.600, \mathrm{p}=0.004)$ than those in the " $\$ 60,000$ to $\$ 99,999 "(\mathrm{~m}=5.913, \mathrm{SE}=1.715, \mathrm{p}=0.001)$ and in the "less than $\$ 60,000 "(m=5.163, \mathrm{SE}=1.918, \mathrm{p}=0.399)$ income groups, although the latter did not reach significance following Bonferroni correction. Children with older parents $(45+)(\mathrm{m}=2.374, \mathrm{SE}=1.808)$ had significantly lower anxiety mean scores $(\mathrm{F}(2,194)=4.849, \mathrm{p}=0.009)$ than children with parents aged 35-44 $(\mathrm{m}=6.317, \mathrm{SE}=1.883, \mathrm{p}<0.001)$ and 18-34 $(\mathrm{m}=6.417, \mathrm{SE}=1.895, \mathrm{p}<0.001)$ years. Children younger than 5 years $(\mathrm{m}=.502, \mathrm{SE}=1.141)$ had significantly lower mean anxiety scores $(\mathrm{F}(3,194)=8.19$, $\mathrm{p}<0.001)$ compared to children aged $6-9(\mathrm{~m}=7.458, \mathrm{SE}=1.749, \mathrm{p}<0.001)$, $10-13(\mathrm{~m}=6.582, \mathrm{SE}=1.733, \mathrm{p}<0.001)$ and $14-18(\mathrm{~m}=5.602$, $\mathrm{SE}=1.868, \mathrm{p}<0.001)$ years. For a change in household 
Table 3 Pairwise comparisons of factors impacting child anxiety $(\mathrm{N}=217)$ and depression $(\mathrm{N}=212)$ in univariate general linear models with model-adjusted estimated marginal means ${ }^{\text {a }}$

\begin{tabular}{|c|c|c|c|c|}
\hline & GAD-7 Mean (SE) & Main Effect ${ }^{b}$ & PHQ-9 Mean (SE) & Main Effect ${ }^{b}$ \\
\hline Parent Age & & $\mathrm{F}(2,194)=4.85^{* *}$ & & $\mathrm{~F}(2,189)=6.04 * *$ \\
\hline $18-34$ & $6.417(1.895)$ & & $6.335(2.355)$ & \\
\hline $35-44$ & $6.317(1.883)$ & & $4.79(2.342)$ & \\
\hline $45+$ & $2.374(1.808)$ & & $0.300(2.237)$ & \\
\hline Child Age & & $\mathrm{F}(3,194)=8.19 * * *$ & & $\mathrm{~F}(3,189)=16.28 * * *$ \\
\hline $0-5$ & $0.502(2.141)$ & & $-3.827(2.661)$ & \\
\hline $6-9$ & $7.458(1.749)$ & & $6.956(2.176)$ & \\
\hline $10-13$ & $6.582(1.733)$ & & $6.172(2.15)$ & \\
\hline $14-18$ & $5.602(1.868)$ & & $5.936(2.318)$ & \\
\hline Household Income (CAD) & & $\mathrm{F}(2,194)=5.60 * *$ & & $\mathrm{~F}(1,189)=4.87 * *$ \\
\hline$<\$ 60,000$ & $5.163(1.918)$ & & $4.318(2.398)$ & \\
\hline$\$ 60,000-\$ 99,999$ & $5.913(1.715)$ & & $4.618(2.130)$ & \\
\hline$>\$ 100,000$ & $4.031(1.732)$ & & $2.492(2.152)$ & \\
\hline Change in household employment & & $F(3,194)=2.655$ & & $F(3,189)=0.740$ \\
\hline Both parents lost work hours & $6.197(1.736)$ & & $4.310(2.160)$ & \\
\hline Participant parent lost work hours & $4.939(1.813)$ & & $4.066(2.259)$ & \\
\hline Spouse lost work hours & $4.701(1.807)$ & & $3.736(2.249)$ & \\
\hline Neither parent lost work hours & $4.307(1.813)$ & & $3.125(2.247)$ & \\
\hline Child Pre-Existing Psychiatric Condition (Anxiety) & & $\mathrm{F}(1,194)=0.004$ & & $F(1,189)=0.125$ \\
\hline Yes & $5.066(1.990)$ & & $3.596(2.484)$ & \\
\hline No & $5.006(1.591)$ & & $4.022(1.968)$ & \\
\hline Child Pre-Existing Psychiatric Condition (Depression) & & $F(1,194)=0.170$ & & $F(1,189)=0.073$ \\
\hline Yes & $5.323(2.198)$ & & $4.044(2.740)$ & \\
\hline No & $4.749(1.474)$ & & $3.574(1.822)$ & \\
\hline Child Pre-Existing Psychiatric Condition (Other) & & $\mathrm{F}(1,194)=1.483$ & & $F(1,189)=0.527$ \\
\hline Yes & $4.536(1.952)$ & & $3.433(2.439)$ & \\
\hline No & $5.536(1.600)$ & & $4.185(1.978)$ & \\
\hline
\end{tabular}

${ }^{a}$ Means presented are based on the estimated marginal means of the model. Bonferroni Correction applied as appropriate for multiple comparisons in individual factor ANOVA tests for subgroup differences.

${ }^{b}$ Main effect of each factor presented as F-statistic [degrees of freedom(factor), degrees of freedom(error)]

CAD $=$ Canadian Dollar

$\mathrm{GAD}=$ Generalized Anxiety Disorder Scale

PHQ = Patient Health Questionnaire (depression scale)

$\mathrm{SE}=$ Standard Error

$* \mathrm{p}<0.05$

$* * \mathrm{p}<0.01$

$* * * \mathrm{p}<0.001$

employment $(\mathrm{F}(3,194)=2.655, \mathrm{p}=0.0504)$ the interaction was decomposed since the value approached significance. In families where both parents reported reduced work hours $(m=6.197, \mathrm{SE}=1.736)$, there was a higher child anxiety score than in those with parents who both reported no change in work hours $(\mathrm{m}=4.307, \mathrm{SE}=1.813, \mathrm{p}=0.042)$ although this difference was no longer present following Bonferroni correction $(\mathrm{p}=0.127)$. Pre-existing child anxiety, depression, or other psychiatric illness had no impact on child anxiety scores.
Thus, we may infer that the children aged 5+, with parents aged 18-44, or whose parents reported household incomes lower than $\$ 100,000$, were vulnerable to having anxiety symptoms during quarantine.

\section{Factors Impacting Depression During Quarantine}

Regression modelling was performed with grouping variables outlined in 2.3.2 for parent and child depression scores. Pre-existing child psychiatric conditions were 
grouped into a single yes/no variable for parent analyses, and parent psychiatric conditions were grouped for child analyses. For factors involving 3 or more groups, Bonferroni corrections for multiple comparisons were used and the resulting $\mathrm{p}$-value is presented for within-group comparisons.

\section{Parental Depression}

Based on the model, the estimated marginal mean for parent depression was $8.673(\mathrm{SE}=1.885)$. Factors that significantly impacted the parents' depression scores were parent and child age, parent sex, and pre-existing psychiatric conditions (not anxiety and depression) [Table 2].

While household income was not a significant predictor $(\mathrm{F}(2,184)=3.007, \mathrm{p}=0.052)$, parent depression scores increased as household income level decreased. Parents with household incomes of $\$ 60,000$ to $\$ 99,999$ ( $\mathrm{m}=9.401$, $\mathrm{SE}=1.869$ ) had higher depression scores than those with household incomes $>\$ 100,000(\mathrm{~m}=7.708, \mathrm{SE}=1.934)$, and also higher than those with household incomes of less than $\$ 60,000(\mathrm{~m}=8.909, \mathrm{SE}=2.084)$. Parents' depression scores significantly decreased with age $(\mathrm{F}(2,184)=8.375$, $\mathrm{p}<0.001)$; specifically, those $45+(\mathrm{m}=4.645, \mathrm{SE}=2.084)$ had significantly lower depression scores than those between 35-44 $(\mathrm{m}=9.769, \mathrm{SE}=2.006, \mathrm{p}<0.001)$ and those between 18-34 $(\mathrm{m}=11.604, \mathrm{SE}=2.070, \mathrm{p}<0.001)$. Females $(\mathrm{m}=9.876, \mathrm{SE}=1.982)$ had significantly higher depression scores than males $(\mathrm{m}=7.469, \mathrm{SE}=1.844, \mathrm{~F}(1,184)$, $\mathrm{p}<0.001)$. Parents who reported pre-existing psychiatric conditions (not anxiety or depression) had higher depression scores $(m=9.878, \mathrm{SE}=2.010)$ than those who reported no pre-existing psychiatric conditions $(\mathrm{m}=7.467$, $\mathrm{SE}=1.876, \mathrm{~F}(1,184)=6.393, \mathrm{p}=0.12)$. Furthermore, the child's age was a significant predictor of parent depression scores $(\mathrm{F}(3,184)=2.731, \mathrm{p}=0.045)$. Parents with children aged 6-9 had the highest PHQ-9 scores $(m=10.250$, $\mathrm{SE}=1.927)$, followed by parents of teenagers 14-18 $(\mathrm{m}=9.119, \mathrm{SE}=2.140)$, then those with children aged 10-13 $(\mathrm{m}=8.896, \mathrm{SE}=1.902)$, and finally the lowest were parents with children aged $0-5(\mathrm{~m}=6.426, \mathrm{SE}=2.345)$. The parents of children aged 6 to 9 had significantly higher scores on the PHQ than those with children aged 10-13 ( $\mathrm{p}=0.028)$ and those aged 0-5 $(\mathrm{p}=0.041)$. Household income was not a predictor for parental depressive symptoms, nor was a change in household employment, or pre-existing parental anxiety or depression.

Therefore, we may infer that the parents who are aged between 18-44, female, have household incomes between $\$ 60,000$ to $\$ 99,999$, have pre-existing psychiatric conditions (not anxiety or depression), or who had children aged 6-9 were more vulnerable to experiencing depressive symptoms during quarantine.

\section{Child Depression}

Based on the model, the estimated marginal mean for child depression was 3.809 ( $\mathrm{SE}=2.159)$. Factors significantly impacting children's depression scores were household income, parent age and child age [Table 3].

Children with household incomes $>\$ 100,000$ had lower depression scores $(m=2.493, S E=2.152)$ $(\mathrm{F}(2,189)=4.870, \mathrm{p}=0.009)$ than those with $\$ 60,000$ to $\$ 99,999(\mathrm{~m}=4.618, \mathrm{SE}=2.130, \mathrm{p}<0.001)$ and $<\$ 60,000$ $(\mathrm{m}=4.318, \mathrm{SE}=2.398, \mathrm{p}=0.468)$ although the latter did not reach significance following Bonferroni correction. Children with parents aged $45+(\mathrm{m}=0.300$, $\mathrm{SE}=2.237)$ had significantly lower depression scores $(\mathrm{F}(2,189)=6.040, \mathrm{p}=0.003)$ than those with parents aged 35-44 $(\mathrm{m}=4.793, \mathrm{SE}=2.342, \mathrm{p}<0.001)$ and $18-34$ $(\mathrm{m}=6.335, \mathrm{SE}=2.355, \mathrm{p}<0.001)$. Children in the 0.5-5 age group $(\mathrm{m}=-3.827, \mathrm{SE}=2.661)$ had significantly lower depression scores $(\mathrm{F}(3,189)=16.285, \mathrm{p}<0.001)$ than children aged 6-9 $(\mathrm{m}=6.956, \mathrm{SE}=2.176, \mathrm{p}<0.001), 10-13$ $(\mathrm{m}=6.172, \mathrm{SE}=2.150, \mathrm{p}<0.001)$ and $14-18(\mathrm{~m}=5.936$, $\mathrm{SE}=2.318, \mathrm{p}<0.001)$ years. A change in parental employment, and pre-existing child psychiatric conditions did not affect the child's reported depression score.

Thus, we may infer that the children aged 6 and older, with parents younger than 45, or those living in households earning less than $\$ 100,000$ were more vulnerable to depression symptoms during quarantine.

\section{Factors Impacting Family Functioning During Pandemic}

Mean family functioning scores were obtained by taking the average of the parent's and child's scores on the FAD $(m=2.249, \mathrm{SD}=.405)$. The binary logistic regression model was fitted with all grouping variables and proceeded with likelihood ratio backward-selection. The factors that significantly impact the odds of having problematic family functioning were parent age, change in household employment status, and pre-existing medical conditions in children. The following odds ratios were significant at the $(\mathrm{p}<0.05)$ level. The model suggests a significant decrease in odds where there are no children with pre-existing medical conditions $(\mathrm{OR}=0.275,95 \% \mathrm{CI}$ $[0.094,0.803])$, or where neither parent lost work hours $(\mathrm{OR}=0.242,95 \% \mathrm{CI}[0.081,0.719])$. Furthermore, having a parent aged 18-34 is associated with a significant increase in likelihood of having problematic family functioning $(\mathrm{OR}=5.046,95 \% \mathrm{CI}[1.092,23.329])$ compared to older age groups. Child age and pre-existing psychiatric conditions significantly impacted family functioning scores; however, problematic family functioning did not significantly vary within those categories [Table 4]. 


\section{Discussion}

This cross-sectional study of Canadian families is among the first to document factors that influence mental health of both adult and child family members and family functioning during the COVID-19 pandemic.

In this sample $78.5 \%$ of families surveyed met the threshold mean of $\geq 2 / 4$ on the FAD-GF indicating problematic family functioning. Many parents experienced anxiety (62.9\%) and depression (73.4\%) symptoms above the threshold for clinical concern for these illnesses (Löwe et al., 2008; Manea et al., 2012). Many children were also above threshold scores for anxiety (54.6\%) and depression (70.7\%).

Whereas certain factors that influence anxiety and depression are similar to those reported elsewhere (Vindegaard \& Benros, 2020; Salari et al., 2020; Luo et al., 2020; Pierce et al., 2020) including parent age, parent gender, socioeconomic status, and pre-existing medical and psychiatric conditions in adults and children, we report several important considerations particular to this family sample. Despite a high proportion of participants reporting a loss of work hours or employment, many families did not report a change in family household income. Some of these families may have received the Canadian Emergency Response Benefit, a $\$ 2000$ monthly payment to offset income and employment changes, offsetting income loss. A higher proportion of children and parents with psychiatric and medical illness is present in this sample likely due to recruitment via parental, family, and disease-specific support groups.
Mean scores in this sample on the FAD-GF above threshold suggest poor family functioning in most families, which may be due to or worsened by the pandemic and quarantine measures. With over $75 \%$ of families meeting the threshold indicative of poor family functioning, it is important to explore possible influencing factors. Specifically, the model for family functioning found that parent age, child age, change in employment, and pre-existing child psychiatric and medical conditions impacted mean family functioning scores. The youngest group of parents (18-34) had roughly 5 times the odds of problematic family functioning compared to the oldest group (45+). Older parents may have developed more coping skills and adaptive strategies, better equipping them for stressors brought about by drastic life changes than their younger counterparts (Carstensen, 2006; Neubauer et al., 2019; Vahia et al., 2020) .

Medical and psychiatric illnesses in a family may impact the likelihood of poor family functioning. Families having members with pre-existing conditions may have developed resilience pathways, but these may also be overwhelmed by the pandemic worsening family dysfunction (Friedmann et al., 1997; Tan et al., 2019). Caregiver wellbeing is an important mediator of healthy parenting and absence of psychiatric illness reduces the strain placed on caregiving parents (Sprang \& Silman, 2013; Friedmann et al., 1997). Families whose child(ren) had a chronic medical illness were more likely to have poor family functioning. The increased parental worry for the health of their child may contribute to illness anxiety and increase their caregiving burden. This parental disruption

Table 4 Binary logistic regression analyses of factors impacting family functioning $(\mathrm{N}=223)$

\begin{tabular}{|c|c|c|c|c|c|}
\hline Final Model & Wald & p-value & Odds Ratio & Lower Bound & Upper Bound \\
\hline \multicolumn{6}{|l|}{ Parent Age* } \\
\hline$(45+)$ & 7.234 & 0.027 & & & \\
\hline$(18-34)$ & 4.293 & 0.038 & 5.046 & 1.092 & 23.329 \\
\hline$(35-44)$ & 0.397 & 0.528 & 1.526 & 0.410 & 5.673 \\
\hline \multicolumn{6}{|l|}{ Child Age* } \\
\hline$(14-18)$ & 9.007 & 0.027 & & & \\
\hline$(0.5-5)$ & 2.605 & 0.107 & 0.224 & 0.036 & 01.378 \\
\hline$(6-9)$ & 2.180 & 0.140 & 0.392 & 0.113 & 1.359 \\
\hline$(10-13)$ & 0.216 & 0.642 & 1.341 & 0.389 & 1.359 \\
\hline \multicolumn{6}{|l|}{ Change in household employment* } \\
\hline Both parents lost work hours & 6.535 & 0.088 & & & \\
\hline Participant parent lost work hours & 3.131 & 0.077 & 0.334 & 0.099 & 1.125 \\
\hline Spouse lost work hours & 2.786 & 0.095 & 0.356 & 0.106 & 1.197 \\
\hline Neither parent lost work hours & 6.518 & 0.011 & 0.242 & 0.081 & 0.719 \\
\hline Child Pre-existing medical condition (no)* & 5.572 & 0.018 & 0.275 & 0.094 & 0.803 \\
\hline Child Pre-existing psychiatric condition (no)* & 3.248 & 0.071 & 2.5527 & 0.922 & 6.927 \\
\hline
\end{tabular}

*All factors presented significantly contributed to the model for impaired family functioning. For factors with $>2$ groups, the factor without a reported odds ratio is held constant for comparison to other groups 
may influence marital and parent-child relationships, sharing these worries with other family members and consequently worsening family functioning (Sprang \& Silman, 2013; Pierce et al., 2020; Tso et al., 2020).

Taxing conditions inherent to the pandemic and lockdown measures may result in or exacerbate existing anxious and depressive symptoms present in children and parents. These symptoms can serve as a proxy for worsening family functioning since they not only impact family units directly, but also the parent-child relationships (Prime et al., 2020). This bi-directional interaction can include erosion of caregiving capabilities and increased strain on parents due to worsening mental health of parents and children respectively (Ashikkali et al., 2020).

Several factors in anxiety and depression models mirror those that were significant in the family functioning model. Parental age was significant in all models, with the oldest parents $(45+)$ being associated with significantly lower anxiety and depression scores in parents and their children. Beyond possessing protective coping strategies, older parents may have fewer pre-pandemic negative emotions allowing them to better respond to their children's needs (Löwe et al., 2010). The youngest children (0-5) had GAD-7 and PHQ-9 scores significantly lower than all other age groups. These children would have experienced the least disruption to their daily routines (no school, time spent mostly with caregivers) due to the pandemic. Parents with pre-existing psychiatric conditions also reported higher anxiety scores, even in those with other non-anxiety conditions. Uncertain and sometimes conflicting information about quarantine measures and infection risk may exacerbate present mental illness symptoms and create difficult family environments, worsening anxious and depressive symptoms.

Female parent respondents experienced significantly higher levels of anxiety and depression, mirroring findings from other studies and reviews (Salari et al., 2020; Özdin \& Bayrak Özdin, 2020; Pierce et al., 2020). Annual household income above $\$ 100,000$ was significantly associated with less anxiety and depression in parents and children. In addition to financial security, elevated household income may confer more resources for mitigating the negative effects of physical and social isolation due to pandemic quarantine measures.

The factors of marital status, ethnicity, change in household income due to COVID-19 and child gender did not impact the mental health or family functioning of our sample.

\section{Limitations}

This study is not without limitations. The online selfreport survey format necessitated by the pandemic prevents independent verification of information. Additionally, the cross-sectional nature of the study prevents causal inferences from being made. The small sample size for a crosssectional sample did not allow for sufficient subgroup sizes to assess all possible associations (e.g. interactions between factors).

Sampling and selection bias may have resulted in overrepresentation of certain groups. Recruitment via family support groups may increase the proportion of reported medical and psychiatric illness, and respondents could have been those more severely affected by the pandemic, more available time to complete the study due to reduced work hours, or those pre-occupied with their physical/ mental health. Models included these factors to account for the resulting impact on outcome measures, but they inconsistently contributed to modelling for anxiety, depression, and family functioning. The within-group effects confirm previous studies' findings and contribute to considerations for the impact of the pandemic on families' mental wellbeing.

We only collected data concerning the most-affected child in the household, limiting the generalizability of our results for the general child and adolescent populations. The GAD-7 and PHQ-9, which were used to assess child anxiety and depressive symptoms, are not validated for children under 12. We prompted parents to assist younger children in filling out these questionnaires which would provide some impression of the anxiety and depression impact on younger children.

Finally, the current study did not capture potentially positive perceptions of quarantine that families may have held. These perceptions may have had positive impacts on family functioning that have not been discussed in this paper. Future studies should consider the mediating effects of positive quarantine perceptions and outcomes on family functioning and mental health.

\section{Conclusion}

While isolation measures are beneficial overall in managing a pandemic, the mental health impact of these measures on individuals and families must be considered. This study explored factors associated with reduced psychological wellbeing in a Canadian sample examining both children and parents in families, identifying several factors that contribute to worsening anxiety and depression symptoms and impaired family functioning. Family functioning was associated with parent and child age, change in parent employment, and pre-existing child medical and psychiatric conditions. Specifically, younger parents, older children, loss of work hours by both parents, and pre-existing child medical conditions contributed to 
greater odds of poor family functioning. Elevated parental anxiety and depression were mediated by younger parental age, female sex, annual household income $<\$ 100,000$, and the presence of pre-existing parent psychiatric conditions. Elevated child anxiety and depression were mediated by younger parent age, older child age, and household income $<\$ 100,000$. These symptoms can precipitate worsening family environments by increasing individuals' stress and straining inter-parental and parent-child relationships. This study contributes significantly to the literature on the impact of COVID-19 and isolation measures on Canadian families, identifying factors [outlined above] that may place families at risk of decreased mental wellbeing and pointing to needed interventions to mediate these effects and improve functioning of parents, children and families.

Authors' Contributions All authors contributed to the study conception and design. Material preparation, data collection and analysis were performed by Philippe Hwang, Lara Ipekian and Nikhil Jaiswal. The first draft of the manuscript was written by Philippe Hwang, Lara Ipekian and Nikhil Jaiswal, and all authors commented on previous versions of the manuscript. All authors read and approved the final manuscript.

Funding Information This work was supported by the McGill Interdisciplinary Initiative in Infection and Immunology (MI4)'s ERCF (Round 2) fund (grant number ERCF-R2-52). The authors did not receive any financial support for the research, authorship, and/or publication of this article. The funding agency did not have any role in designing or conducting the study; data collection, analysis, and interpretation; or preparation or approval of the manuscript.

Data Availability Data from this study is not publicly available due to identifying information being accessible via demographic and socioeconomic data. However, the authors will respond to data queries upon request.

\section{Code Availability Not applicable}

\section{Declarations}

Conflicts of Interest/Competing Interests The authors declare only that the study received funding via the MI4 ERCF fund to conduct the research.

Ethics Approval The study was reviewed and approved by the McGill University Health Center Research Institute's Ethics Review Board

Consent to Participate All participants completed a form indicating their consent to participate in this research study prior to completing the questionnaires.

Consent for Publication All participants completed a consent form which included statements about their consent that anonymized data with no identifying information might be used in the production of manuscripts for publication.

\section{References}

Ashikkali, L., Carroll, W., \& Johnson, C. (2020). The indirect impact of COVID-19 on child health. Paediatr Child Health, 30, 430 437. https://doi.org/10.1016/j.paed.2020.09.004

Brooks, S. K., Webster, R. K., Smith, L. E., et al. (2020). The psychological impact of quarantine and how to reduce it: rapid review of the evidence. The Lancet, 395, 912-920. https://doi. org/10.1016/S0140-6736(20)30460-8

Carstensen, L. L. (2006). The Influence of a Sense of Time on Human Development. Science, 312, 1913-1915. https://doi. org/10.1126/science. 1127488

Davidson, B., Schmidt, E., Mallar, C., et al. (2020). Risk and resilience of well-being in caregivers of young children in response to the COVID-19 pandemic. Transl Behav Med. https://doi.org/ $10.1093 /$ tbm/ibaa124

Epstein, N. B., Baldwin, L. M., \& Bishop, D. S. (1983). THE McMASTER FAMILY ASSESSMENT DEVICE*. $J$ Marital Fam Ther, 9, 171-180. https://doi.org/10.1111/j.1752-0606. 1983.tb01497.x

Evanoff, B. A., Strickland, J. R., Dale, A. M., et al. (2020). WorkRelated and Personal Factors Associated With Mental WellBeing During the COVID-19 Response: Survey of Health Care and Other Workers. J Med Internet Res, 22, e21366. https://doi. org/10.2196/21366

Friedmann, M. S., McDERMUT, W. H., Solomon, D. A., et al. (1997). Family Functioning and Mental Illness: A Comparison of Psychiatric and Nonclinical Families. Fam Process, 36, 357-367. https://doi.org/10.1111/j.1545-5300.1997.00357.x

Kroenke, K., Spitzer, R. L., \& Williams, J. B. W. (2001). The PHQ9: Validity of a brief depression severity measure. J Gen Intern Med, 16, 606-613. https://doi.org/10.1046/j.1525-1497.2001. 016009606.x

Lapierre, L. M., van Steenbergen, E. F., Peeters, M. C. W., \& Kluwer, E. S. (2016). Juggling work and family responsibilities when involuntarily working more from home: A multiwave study of financial sales professionals: INVOLUNTARY TELEWORK AND WORK-FAMILY CONFLICT. J Organ Behav, 37, 804822. https://doi.org/10.1002/job.2075

Li, G., Miao, J., Wang, H., et al. (2020). Psychological impact on women health workers involved in COVID-19 outbreak in Wuhan: a cross-sectional study. J Neurol Neurosurg Psychiatry, 91, 895-897. https://doi.org/10.1136/jnnp-2020-323134

Löwe B, Decker O, Müller S, et al (2008) Validation and Standardization of the Generalized Anxiety Disorder Screener (GAD-7) in the General Population: Med Care 46:266-274. doi: 10.1097/ MLR.0b013e318160d093

Löwe, B., Wahl, I., Rose, M., et al. (2010). A 4-item measure of depression and anxiety: Validation and standardization of the Patient Health Questionnaire-4 (PHQ-4) in the general population. J Affect Disord, 122, 86-95. https://doi.org/10.1016/j.jad. 2009.06.019

Luo, M., Guo, L., Yu, M., et al. (2020). The psychological and mental impact of coronavirus disease 2019 (COVID-19) on medical staff and general public - A systematic review and metaanalysis. Psychiatry Res, 291, 113190. https://doi.org/10.1016/j. psychres.2020.113190

Manea, L., Gilbody, S., \& McMillan, D. (2012). Optimal cut-off score for diagnosing depression with the Patient Health Questionnaire (PHQ-9): a meta-analysis. Can Med Assoc J, 184, E191-E196. https://doi.org/10.1503/cmaj.110829

Markenson, D., \& Redlener, I. (2004). Pediatric Terrorism Preparedness National Guidelines and Recommendations: Findings of an Evidenced-based Consensus Process. Biosecurity Bioterrorism 
Biodefense Strategy Pract Sci, 2, 301-319. https://doi.org/10. 1089/bsp.2004.2.301

Mazza, C., Ricci, E., Biondi, S., et al. (2020). A Nationwide Survey of Psychological Distress among Italian People during the COVID19 Pandemic: Immediate Psychological Responses and Associated Factors. Int J Environ Res Public Health, 17. https://doi.org/10. 3390/ijerph17093165

Miller RG (1966) Simultaneous statistical inference, 2nd ed. Springer, New York, NY

Miller, I. W., Epstein, N. B., Bishop, D. S., \& Keitner, G. I. (1985). The mcmaster family assessment device: reliability and validity*. J Marital Fam Ther, 11, 345-356. https://doi.org/10.1111/j.17520606.1985.tb00028.x

Miller, J. J., Cooley, M. E., \& Mihalec-Adkins, B. P. (2020). Examining the Impact of COVID-19 on Parental Stress: A Study of Foster Parents. Child Adolesc Soc Work J C A, 1-10. https://doi.org/10. 1007/s10560-020-00725-w

Neubauer, A. B., Smyth, J. M., \& Sliwinski, M. J. (2019). Age Differences in Proactive Coping With Minor Hassles in Daily Life. $J$ Gerontol Ser B, 74, 7-16. https://doi.org/10.1093/geronb/gby061

Nicholas, D. B., Gearing, R. E., Koller, D., et al. (2008). Pediatric epidemic crisis: Lessons for policy and practice development. Health Policy, 88, 200-208. https://doi.org/10.1016/j.healthpol. 2007.11.006

Özdin, S., \& Bayrak Özdin, Ş. (2020). Levels and predictors of anxiety, depression and health anxiety during COVID-19 pandemic in Turkish society: The importance of gender. Int J Soc Psychiatry, 66, 504-511. https://doi.org/10.1177/0020764020927051

Pierce, M., Hope, H., Ford, T., et al. (2020). Mental health before and during the COVID-19 pandemic: a longitudinal probability sample survey of the UK population. Lancet Psychiatry, 7, 883-892. https://doi.org/10.1016/S2215-0366(20)30308-4

Prime, H., Wade, M., \& Browne, D. T. (2020). Risk and resilience in family well-being during the COVID-19 pandemic. Am Psychol, 75, 631-643. https://doi.org/10.1037/amp0000660

Salari, N., Hosseinian-Far, A., Jalali, R., et al. (2020). Prevalence of stress, anxiety, depression among the general population during the COVID-19 pandemic: a systematic review and meta-analysis. Glob Health, 16, 57. https://doi.org/10.1186/s12992-020-00589-w

Spitzer, R. L., Kroenke, K., Williams, J. B. W., \& Löwe, B. (2006). A Brief Measure for Assessing Generalized Anxiety Disorder: The GAD-7. Arch Intern Med, 166, 1092. https://doi.org/10.1001/archi nte.166.10.1092
Sprang, G., \& Silman, M. (2013). Posttraumatic Stress Disorder in Parents and Youth After Health-Related Disasters. Disaster Med Public Health Prep, 7, 105-110. https://doi.org/10.1017/dmp.2013.22

Staccini, L., Tomba, E., Grandi, S., \& Keitner, G. I. (2015). The Evaluation of Family Functioning by the Family Assessment Device: A Systematic Review of Studies in Adult Clinical Populations. Fam Process, 54, 94-115. https://doi.org/10.1111/famp.12098

Stark, A. M., White, A. E., Rotter, N. S., \& Basu, A. (2020). Shifting from survival to supporting resilience in children and families in the COVID-19 pandemic: Lessons for informing U.S. mental health priorities. Psychol Trauma Theory Res Pract Policy, 12, S133-S135. https://doi.org/10.1037/tra0000781

Tan, J. H. P., Conlon, C., Tsamparli, A., et al. (2019). The association between family dysfunction and admission to an acute mental health inpatient unit: a prospective study. Ir J Psychol Med, 1-11. https://doi.org/10.1017/ipm.2019.41

Torales, J., O'Higgins, M., Castaldelli-Maia, J. M., \& Ventriglio, A. (2020). The outbreak of COVID-19 coronavirus and its impact on global mental health. Int J Soc Psychiatry, 66, 317-320. https:// doi.org/10.1177/0020764020915212

Tso, W. W. Y., Wong, R. S., Tung, K. T. S., et al. (2020). Vulnerability and resilience in children during the COVID-19 pandemic. Eur Child Adolesc Psychiatry. https://doi.org/10.1007/ s00787-020-01680-8

Vahia, I. V., Jeste, D. V., \& Reynolds, C. F. (2020). Older Adults and the Mental Health Effects of COVID-19. JAMA, 324, 2253 https://doi.org/10.1001/jama.2020.21753

Vindegaard, N., \& Benros, M. E. (2020). COVID-19 pandemic and mental health consequences: Systematic review of the current evidence. Brain Behav Immun, 89, 531-542. https://doi.org/10. 1016/j.bbi.2020.05.048

Wang, C., Pan, R., Wan, X., et al. (2020). A longitudinal study on the mental health of general population during the COVID-19 epidemic in China. Brain Behav Immun, 87, 40-48. https://doi.org/ 10.1016/j.bbi.2020.04.028

Zhang, W.-R., Wang, K., Yin, L., et al. (2020). Mental Health and Psychosocial Problems of Medical Health Workers during the COVID-19 Epidemic in China. Psychother Psychosom, 89, 242250. https://doi.org/10.1159/000507639

Publisher's Note Springer Nature remains neutral with regard to jurisdictional claims in published maps and institutional affiliations. 